\title{
VLF Disturbances Caused by Trapped Beta-Rays From the Decay of Neutrons Produced in High-Altitude Nuclear Explosions
}

\author{
A. J. Zmuda, B. W. Shaw, and C. R. Haave
}

\author{
Contribution From Applied Physics Laboratory, The Johns Hopkins University, Silver Spring, Md.
}

\begin{abstract}
In the fall of 1962 nuclear devices exploded near Johnston Island on October 20 and 26 and November 1 produced phase perturbations of very-low-frequency radio waves monitored at APL/JHU. For the event of October 26, and in regard to the VLF transmission from NBA in Balboa, Panama, as received at APL, the perturbation has a temporal variation attributable to trapped $\beta$-rays from the radioactive decay of neutrons. The artificial belt of $\beta$-rays forms around the geomagnetic meridian through the burst point. As the stream drifts eastward in its first global circuit an energy dispersion occurs because the more energetic particles also have a larger azimuthal drift velocity. The attendant perturbations along VLF propagation paths remote from the detonation area have a temporal variation in accord with that of the energy of the trapped $\beta$-ray stream passing over the VLF path. The work on this event confirms the results of an earlier study which contained an uncertainty due to the presence of a natural disturbance, a disturbance which apparently did not especially affect the region herein treated. The VLF perturbations due to the bursts of October 20 and November 1 were relatively small but the former event did produce a disturbance also consistent with the idea that the cause resides in trapped $\beta$-rays from the decay of neutrons.
\end{abstract}

\section{Introduction}

Nuclear explosions above the earth's surface produce a variety of ionospheric disturbances, including modifications in the velocity of propagation of a VLF (very-low-frequency, $3-30 \mathrm{kc} / \mathrm{s}$ ) radio signal. At propagation paths exposed to direct electromagnetic and particle radiation from the burst the VLF perturbations appear practically instantaneous with the burst and stem primarily from X-rays, $\gamma$-rays, and $\beta$-rays [Crain and Tamarkin, 1961 ; Jean and Crombie, 1962 ; Latter, Herbst, and Watson, 1961]. At points sufficiently distant to be screened from the direct burst-effects by the earth and its atmosphere, the VLF disturbances mav be sudden and occur within a few seconds of the detonation, or delayed and occur minutes or tens of minutes after the explosion.

The sudden remote VLF disturbances were first considered by Crain and Tamarkin [1961] who proposed as the ionizing agents $\beta$-rays from the radioactive decay of neutrons: the neutrons from the burst are unaffected by the geomagnetic field, can travel large distances in times of a few seconds, and can deposit their decay products on field lines that channel the charged but essentially untrapped particles, particularly the $\beta$-rays, into the VLF propagation region where they produce ionization.

This work was supported by the Bureau of Naval Weapons, Department of the Navy; under Contract NOw 62-0604-c and by the Geophysics Research Directorate, Air Force Cambridge Research Laboratories.
The delayed remote VLF disturbances stem from an enhancement of atmospheric ionization due to geomagnetically trapped particles which drift from the burst region to the VLF propagation path [Zmuda, Shaw, and Haave, 1963a; Haave, Zmuda, and Shaw, 1962; Willard and Kenney, 1963; Woodward and Gassmann, 1963; Zmuda, Shaw, and Haave, 1963b; Riedler, Egeland, Lindquist, Pedersen, 1963].

For delayed VLF perturbations at remote distances, Zmuda, Shaw, and Haave [1963a] may have given the first extensive treatment on VLF disturbances related to an artificial radiation belt. Some characteristics treated were initial formation of the belt, the energy spectrum and azimuthal drift of trapped $\beta$-rays, the energy dispersion in the belt as the particles make their first global orbit, and finally the close temporal correlation between the VLF disturbance and the relative amount of energy of the trapped radiation over the VLF path. The energy spectrum of the $\beta$-rays determines in a sensitive manner the connection between the trapped radiation belt and properties of the VLF disturbance such as the time of onset, maximum, and periods of increase, decrease, and recovery. For the nuclear explosion of July 9, 1962, the trapped particles were $\beta$-rays with a spectrum similar to that from the decay of fission fragments. The satellite data of O'Brien, Laughlin, and Van Allen [1962] constituted the first observation of this trapped belt whose characteristics still are lacking adequate definition [Brown, Hess 
and Van Allen, 1963]. Zmuda, Shaw, and Haave, [1963a] and Woodward and Gassmann, [1963] treated the associated VLF perturbations.

For the nuclear burst of October 26, 1962, the VLF disturbance along the VLF path from station NPM in Hawaii to Anchorage, Alaska, had a chronology which at least suggested the presence of trapped electrons with a spectrum characteristic of that from the radioactive decay of neutrons [Zmuda, Shaw, and Haave, 1963b], a spectrum readily distingushable from that of fission betas. As noted in the referenced publication this early work was clouded by the presence of a natural ionospheric disturbance. In an attempt to expand on the question of neutrondecay betas and the VLF disturbance of October 26 1962 , and to minimize the effect of the natural disturbance, we here consider the VLF perturbation of the signal from station NBA in Balboa, Panama, as received at APL/JHU. This propagation path lies in the main at latitudes considerably lower than those heretofore treated and in an area where the natural effects are in general considerably reduced from those existing at high latitudes. In addition, the VLF disturbances associated with the nuclear detonations of October 20 and November 1 in 1962 are herein treated.

\section{Brief Review of Some Basic Characteristics}

For clarity of the present development it is worthwhile to review briefly some of the basic considerations discussed at greater length in earlier publica- tions. Figure 1 shows the transmitter locations, the receiver sites at APL/JHU and Anchorage, Alaska, and the VLF propagation paths superposed on the contours of the magnetic induction $B$ and of $\mathrm{McIl}-$ wain's $L$ [1961]. The $B-L$ contours are for an altitude of $100 \mathrm{~km}$ and were prepared by the Dasa Data Center/Dasa Computing Center, General Electric Company, Santa Barbara, Calif. Some pertinent station characteristics are listed in table 1 and are later also discussed.

In the mode theory of VLF propagation (Wait, 1959 and 1963) the signal is considered to propagate in the spherical waveguide formed by the earth's surface and the lower ionosphere whose effective reflecting height varies between 70 and $90 \mathrm{~km}$, respectively, for all-daylight and all-darkness conditions over the path. In practice the phases of the stabilized VLF transmissions are measured with respect to a local reference oscillator with the changes frequently described in terms of the relative time-of-arrival of the VLF signal, with arrival-time magnitudes generally of the order of tens of microseconds. (The APL facility is described by Shaw and Haave [1962].) The VLF signal undergoes a normal diurnal variation due to the effects of photo-ionization [Pierce, 1955; Wait, 1959; Blackband, 1963]. After nuclear detonations, VLF phase perturbations often stem from an enhancement of ionization produced by burst-related electrons with sufficient energy to penetrate through the upper atmosphere to the VLF propagation region; considering an altitude of $90 \mathrm{~km}$, the electron energy must exceed $50 \mathrm{kev}$.

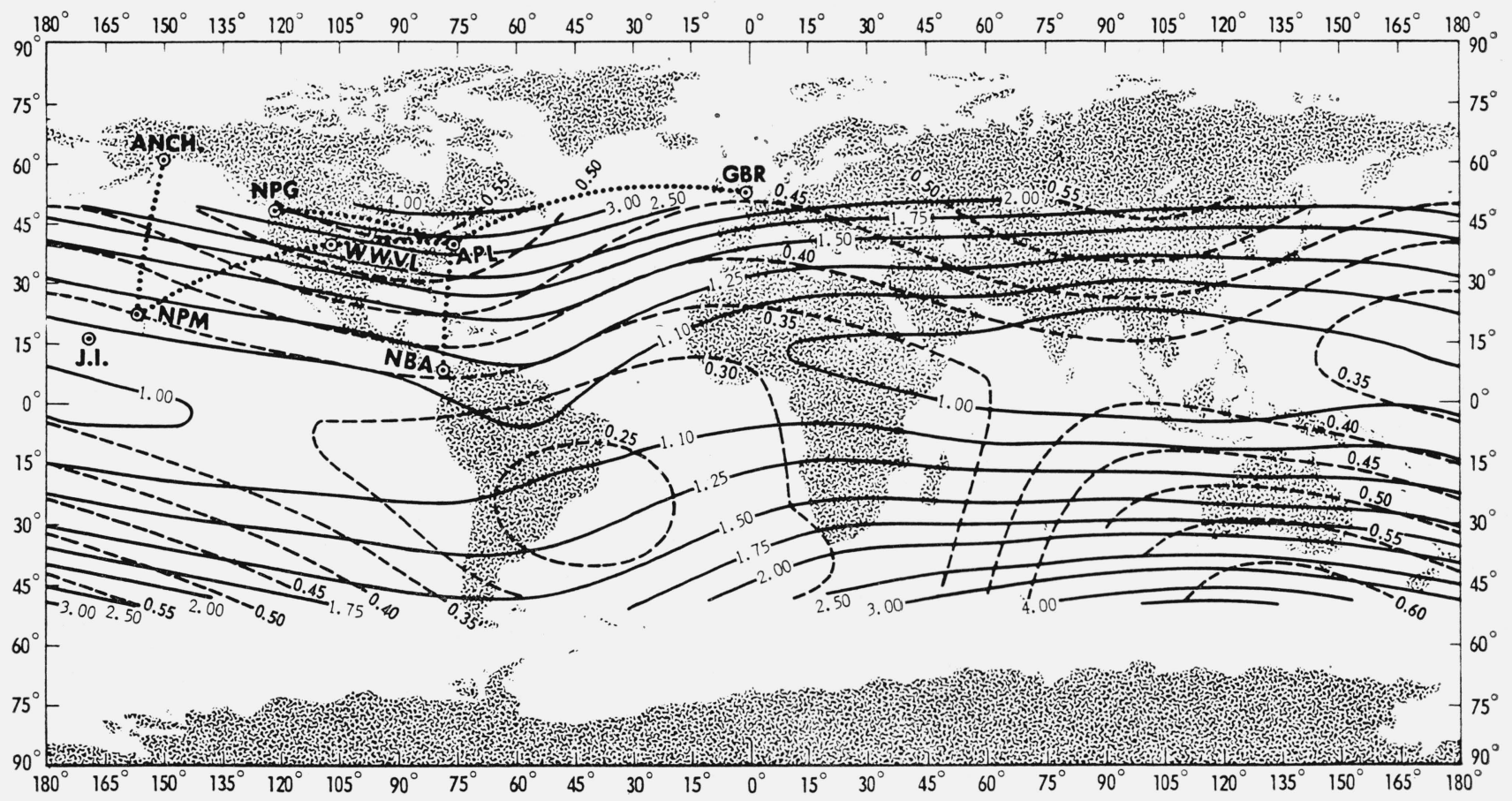

Figure 1. The VLF station locations and propagation paths superposed on the contours of the magnetic induction B, the dashed curves, and of McIlwain's L, the solid curves.

The $B$ and $L$ contours are for an altitude of $100 \mathrm{~km}$ and were prepared by the Dasa Data Center/Dasa Computing Center, General Electric Company, Santa Barbara, Calif. 
TABLE 1. Some characteristics of stations and VLF transmissions

\begin{tabular}{|c|c|c|c|c|c|c|c|}
\hline \multicolumn{2}{|c|}{ Station } & \multirow{2}{*}{$\begin{array}{l}\text { Transmitter } \\
\text { frequency }\end{array}$} & \multirow{2}{*}{$\begin{array}{l}\text { Distance } \\
\text { from } \\
\text { Johnston } \\
\text { Island }\end{array}$} & \multicolumn{2}{|c|}{$\begin{array}{l}\text { Geographic } \\
\text { coordinates }\end{array}$} & \multicolumn{2}{|c|}{$\begin{array}{l}\text { Geomagnetic } \\
\text { coordinates }\end{array}$} \\
\hline Call & Location & & & N. Lat. & W. Long. & N. Lat. & W. Long. \\
\hline $\begin{array}{l}\text { NPG } \\
\text { NBA } \\
\text { WWVL } \\
\text { NPM } \\
\text { APL/JHUUU NOH ISLAND } \\
\text { JOHSTON }\end{array}$ & $\begin{array}{l}\text { Jim Creek, Washington } \\
\text { Balboa, Panama... } \\
\text { Boulder, Colo } \\
\text { Lualualei, Hawaii } \\
\text { Howard County, Md. }\end{array}$ & $\begin{array}{c}\mathrm{kc} / \mathrm{sec} \\
18.6 \\
18 \\
20 \\
19.8\end{array}$ & $\begin{array}{r}k m \\
5500 \\
9600 \\
6600 \\
1400 \\
9100\end{array}$ & $\begin{array}{l}47 \\
09 \\
40 \\
21 \\
39 \\
17\end{array}$ & $\begin{array}{r}123 \\
80 \\
106 \\
158 \\
77 \\
170\end{array}$ & $\begin{array}{l}53 \\
21 \\
49 \\
21 \\
51 \\
14\end{array}$ & $\begin{array}{r}66 \\
12 \\
44 \\
94 \\
10 \\
104\end{array}$ \\
\hline
\end{tabular}

If an artificial radiation belt forms in the vicinity of the detonation, the charged particles drift azimuthally due to the curvature and inhomogeneity of the geomagnetic field [Alfvén, 1948; Spitzer, 1959]. Figure 2 shows the eastward drift velocities for electrons mirroring at $80 \mathrm{~km}$ altitude and for various geomagnetic latitudes computed for the dipole field using the expressions of Hamlin, Karplus, Vik, and Watson [1961]. Though the drift is for altitudes of $80 \mathrm{~km}$, it is essentially unchanged for particles mirroring as high as $1,000 \mathrm{~km}$. Note that (1) for a fixed particle energy, the drift velocity increases with geomagnetic latitude, and (2) for a fixed latitude, the drift velocity increases practically linearly with energy.

About one neutron per fission escapes the bomb device which produces about $1.5 \times 10^{23}$ fissions per kiloton fission explosion [Latter, Herbst, and Watson, 1961]. The free neutron decays with a half-life of about $13 \mathrm{~min}$ into a proton, $\dot{\beta}$-ray, and neutrino; the proton kinetic energies are nearly those of the parent neutrons, which generally have maximums of the order of 1 to $10 \mathrm{Mev}$ [Glasstone, 1962]. The

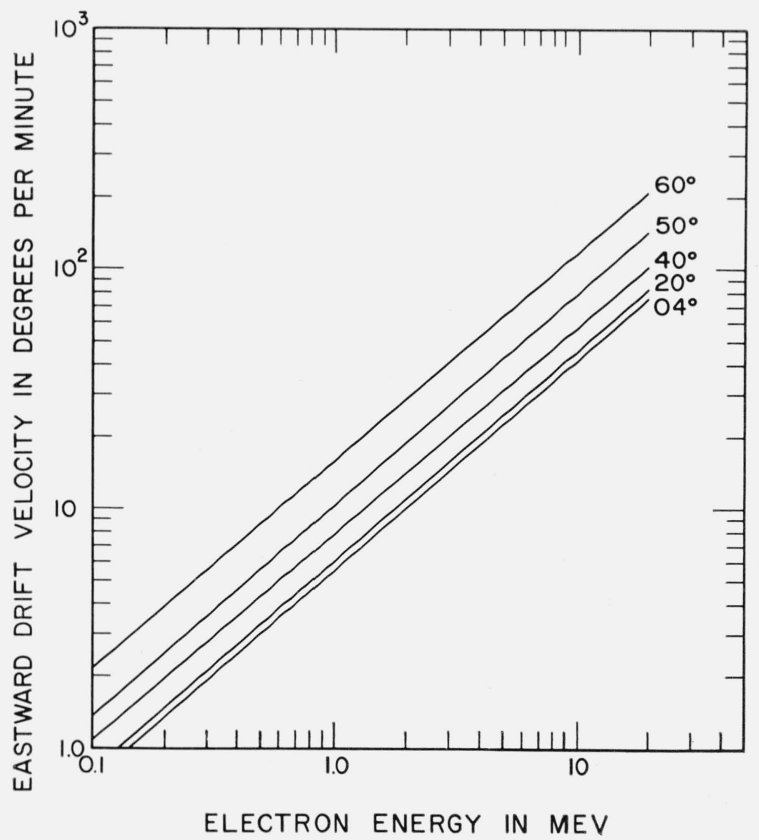

FIgure 2. The longitudinal drift of trapped $\beta$-rays mirroring at an altitude of $80 \mathrm{~km}$ for various geomagnetic latitudes. solid curve in figure 3 depicts the shape of the $\beta$-ray spectrum for the relative number of $\beta$-rays per unit energy interval, from the well-known theory of Fermi as treated in Evans [1955]. The curve has a maximum for a $\beta$-ray of energy $0.25 \mathrm{Mev}$ and an endpoint energy of $0.78 \mathrm{Mev}$, an energy corresponding to that derived from the mass difference between the neutron and the proton. Now the atmospheric ionization, and hence the associated VLF disturbance, depends on the energy deposited at the altitudes of interest (with an electron losing $33 \mathrm{ev}$ of energy for each electron-ion pair created). As a consequence, it is necessary to consider primarily the relative $\beta$-ray kinetic energy per unit interval determined by multiplying the data in the number spectrum by the midpoint of the relevant energy interval. The resultant values are shown in figure 3 as the dashed curve, which has a maximum for $\beta$-rays of energy 0.37 to $0.42 \mathrm{Mev}$.

While the data in figure 3 are for $\beta$-rays from a neutron at rest, the endpoint energy and the major portion of the spectrum, with emphasis on the higher energies, are essentially unchanged for $\beta$-rays from neutrons with energies comparable to those expected from nuclear reactions: adding the neutron velocity relativistically to that of the $\beta$-ray changes the latter only by a relatively small amount. It is important to emphasize that the neutron-decay $\beta$-ray spectrum is drastically different and hence readily distinguishable from that for $\beta$-rays from the decay of fission fragments. Fission betas have, for

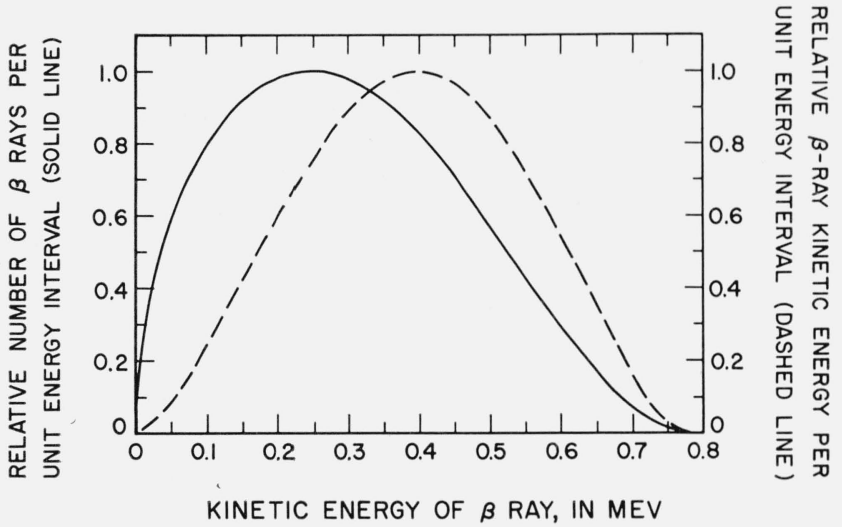

FIGURE 3. Energy spectrum of $\beta$-rays from the radioactive decay of neutrons at rest. 
example, energies extending out to at least $8 \mathrm{Mev}$ and contain large, if not predominant, quantities of particles with energies exceeding the endpoint energy of $0.78 \mathrm{Mev}$ for neutron-decay $\beta$-rays. These fission betas were important in the delayed VLF effects produced after the high-altitude explosion of July 9, 1962 [Zmuda, Shaw, and Haave, 1963a].

\section{NBA-APL Disturbance on October 26, 1962}

Figure 4 shows the diurnal variation of the NBA signal as received at APL/JHU for the Greenwich days of October 24, 25, and 26 in 1962 . (This propagation path lies practically along a geomagnetic meridian.) The daily values are normalized with respect to that existing at $1000 \mathrm{U}$ T, a time which on the burst date of October 26 corresponds to the time of detonation. The total variations for October 24 and 25 are characteristic of the normal, quiet-day changes for this season. During the period $0200 \mathrm{UT}$ to about 1040 U'T the total path lies in darkness, with the variations in signal arrival-time of the order of 1 or $2 \mu \mathrm{sec}$ in magnitude. The sunrise decrease in signal arrival-time (or advance in signal phase) commences at about 1040 U'T and continues until about $1200 \mathrm{U}$ T, with a total decrease in arrival-time of about $40 \mu \mathrm{sec}$. After $1200 \mathrm{UT}$ and while the total path is in daylight, the propagation time stays relatively constant until the sunset phase retardation begins about $2200 \mathrm{U} T$ and ends about $0200 \mathrm{U}$ T on the next day. For the NBA-APL path, through the application of Wait's [1959] first-order theory, a decrease of $1 \mu \mathrm{sec}$ in signal arrival-time (or an advance in signal phase of $6.5^{\circ}$ ) corresponds to a lowering of the effective ionosphere height of about $0.8 \mathrm{~km}$.

On October 26, the VLF variation in the period $0300 \mathrm{U} T$ to $0800 \mathrm{U}$ T is depressed by about $5 \mu$ sec, in relation to the variation for the preceding nights. The direct cause for this deviation is currently unknown, although it is relevant to note that an examination of middle-latitude magnetograms indicate the existence of small ionospheric disturbances during this period. In the preburst period $0900 \mathrm{U}$ T to 1000 U'T, as well as the post-burst period until about 1010

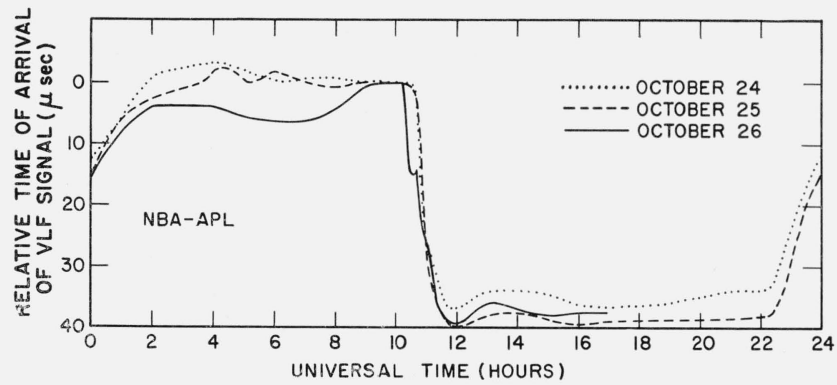

Figure 4. The diurnal changes in the arrival-time of the NBA signal at $18 \mathrm{kc} / \mathrm{s}$, as received at APL/JHU on October 24 , 25 , and 26 in 1962 .

The nuclear detonation occurred at 1000 UT on October 26.
U'T (when the burst-related perturbation begins), the signal arrival-time is essentially constant indicating the absence of any VLF perturbations. The period around the burst time is subsequently discussed in detail and for the present purpose it suffices to add now that from the start of the sunrise transition, at 1040 U'T, until 1700 U'T when APL temporarily discontinued monitoring NBA, the VLF phase changes for October 26 were essentially equal to those found on the undisturbed days, October 24 and 25. It is of prime significance that for the 70min period immediately before the burst-connected VLF disturbance, quiet-day characteristics exist over this propagation path. Thus, the uncertainty associated with the analysis of the NPM to Anchorage disturbance [Zmuda, Shaw, and Haave, 1963b] may be reasonably excluded from the present considerations.

The detailed VLF perturbations occurring in the epoch near the burst are indicated by the solid curve of figure 5a which also contains for comparison the variations for the comparable period on October 25, a characteristic quiet-day variation. In this figure the time $t=0$ corresponds to $1000 \mathrm{UT}$ which, as previously noted, is the burst time on October 26 , 1962. On the day of the nuclear detonation, as previously noted, the VLF propagation time remained practically constant, varying by at most 1 $\mu$ sec in the observation period $-60<t<10 \mathrm{~min}$, a period which contained a normal interruption of the NBA transmission in $4<t<7 \mathrm{~min}$. At about $10 \mathrm{~min}$ after the burst, the signal arrival-time begins a slow

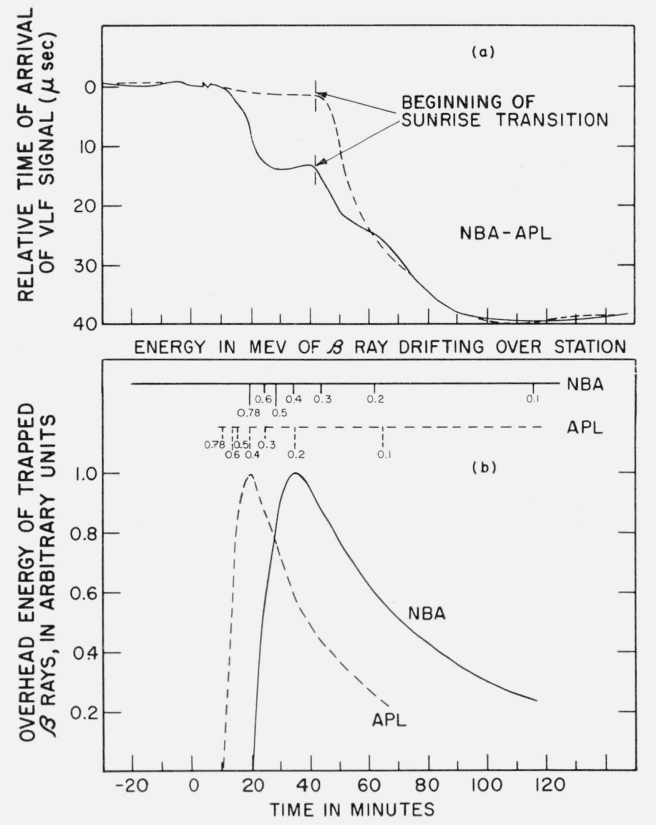

FIGURE 5. The solid curve in the top figure shows the changes in arrival-time of the NBA to $A P L$ transmission for the period around the time of the burst, 1000 UT on October 26,1962, a time taken as $\mathrm{t}=0$.

The dashed curve in the ton tigure indicates a characteristic quiet-day variation for a comparable period. The bottom figure illustrates the temporal change in the energy of trapped neutron-decay $\beta$-rays drifting over NBA and APL, and the the energy of trapped neutron-decay $\boldsymbol{\beta}$-rays drift
times when individual $\boldsymbol{\beta}$-rays are overhead. 
gradual decline with the decrease steepening at about $t=13 \mathrm{~min}$ and reaching a broad flat maximum depression of $14 \mu \mathrm{sec}$ in $27<t<34 \mathrm{~min}$. The signal arrival-time then starts a recovery towards the normal nighttime value until about $t=40$ when sunlight illuminates the propagation path. After the sunrise transition the effects of atmospheric ionization by solar radiation obscure whatever remain of the ionizing effects due to the nuclear burst.

The temporal variations of the VLF signals from NBA to APL are consistent with the model proposed in our earlier work relating to this nuclear event. In general, the delayed VLF disturbance on this date stems from an enhancement of ionization produced in the VLF region by neutron-decay beta rays temporarily trapped in the geomagnetic field. We consider that a belt of these particles forms in the geomagnetic meridian through the burst point. As the stream of $\beta$-rays drifts eastward an energy dispersion occurs because the azimuthal drift rate varies essentially directly with particle energy and, as a consequence, the more energetic particles move faster eastward (in addition to traveling faster along the field lines). Some particles in the drifting stream have pitch angles permitting penetration to the lower ionosphere where, through collisions with the atmospheric constituents, an enhancement of ionization is produced with attendant disturbances such as phase perturbations of VLF signals. Viewed from a fixed point such as one or the other of the terminal points on the NBA-APL path, the overhead energy of the trapped stream changes chronologically in a manner fundamentally dependent on the energy spectrum of the $\beta$-rays.

In addition to placing the radiation belt initially (that is, at $t=0$ ) in the geomagnetic meridian through the burst point, consider also that the latitudinal extent at formation at least encompasses a range equaling that spanning the terminal points of this path. (We later return to the question of the latitudinal and longitudinal width of the belt at formation.) For a trapped stream in its first orbit around the world, figure $5 \mathrm{~b}$ shows the relative amount of the stream energy overhead at NBA and APL at any instant as well as the times when singular $\beta$-rays are over the station. Recalling that for a fixed particle energy the drift velocity increases with latitude, one determines that the first contact of the stream with the VLF path occurs $11 \mathrm{~min}$ after the burst. At this time the $0.78 \mathrm{Mev}$ betas drift across APL, their crossing being practically coincident with the onset of the VLF perturbation. The first contact at lower-latitude points occurs at $t>11$ and culminates in the passage at $t=20 \mathrm{~min}$ of the $0.78 \mathrm{Mev}$ betas over NBA.

Since a decrease in VLF signal arrival-time in the present case corresponds to an increase in the VLF perturbation and, if we defer for the moment consideration of the period $10<t<20 \mathrm{~min}$, the temporal variation of the stream energy over NBA is in accord with that of the VLF disturbance, in the interval $20<t<43 \mathrm{~min}$. Beyond $t=20$, the overhead particle energy and the VLF disturbance both increase to practically coincident maxima and then simultaneously decrease in $37<t<43$, a period after which the burst effects are obscured by the contribution of ionization due to sunlight.

The VLF disturbance in $10<t<20$ undoubtedly results from ionization due to $\beta$-rays drifting at latitudes north of NBA. In an attempt to bracket the latitudinal extent of the trapped radiation, we plot (as previously noted) the overhead energy at APL assuming the absolute amount of this energy, whose magnitude is currently unknown, equals that over NBA. Considering the present data alone, there is no compelling reason for reducing the overhead energy of APL relative to NBA, although there may be a hint for reduction. As discussed earlier, there is good agreement in the period $20<t<43$ between the temporal variation of the VLF disturbance and the overhead stream energy at NBA. For this period this correlation indicates the dominance of the ionization in regions near NBA to the relative exclusion of that for regions north of NBA. Beyond the present data, as pointed out in a previous paper (Zmuda, Shaw, and Haave, 1963b) no VLF perturbation appears in the transmission from station NPG in Jim Creek, Washington, as received at APL a path which lies totally within the geomagnetic latitude range of $51^{\circ}$ to $53^{\circ} \mathrm{N}$. This absence appears to be irreconcilable with the presence of large amounts of trapped radiation at the latitudes of APL or NPG.

In general, the totality of factors at least suggest that in relation to the NBA-APL path, the trapped radiation (and hence the ionization producing the VLF disturbance) decreases in going northward from NBA and is small but significant at APL, significant in view of the simultaneity of the onset of the VLF disturbance and the arrival over APL of the $0.78 \mathrm{Mev}$ betas. (In going southward from NBA, the radiation probably increases until the burst latitude of $14^{\circ}$ geomagnetic is reached, after which it starts decreasing.) With regard to the longitudinal extent of the trapped radiation at the time of the formation of the belt, the geomagnetic width probably has an upper limit of $5^{\circ}$ to $9^{\circ}$. This estimate (which can be readily improved in future work) is derived by multiplying the uncertainty of our time resolution, about a minute, by the drift rate of the $0.78 \mathrm{Mev}$ betas at the latitudes of NBA and APL, respectively 4.7 and $8.5 \mathrm{deg}$ per minute. (It is, of course, obvious that the longitudinal extent of the belt broadens with time after the burst, and if the particle lifetimes are sufficiently long, the trapped radiation ultimately encircles the world.)

It is significant to note that the VLF perturbation along the NBA-APL path has temporal variations discordant with those that would have been produced if the trapped particles were $\beta$-rays from the radioactive decay of fission fragments, $\beta$-rays whose energy spectrum differs considerably from that of neutrondecay betas. For example, fission betas have energies extending to at least $8 \mathrm{Mev}$, considerably in excess of the endpoint energy, $0.78 \mathrm{Mev}$, from a $\beta$-ray from the radioactive decay of neutrons. Fission 
betas dominated in the artificial belt formed after the high-altitude nuclear explosion of July 9, 1962, and produced a delayed VLF disturbance along the same NBA-APL path herein discussed [Zmuda, Shaw, and Haave, 1963a]. Applying the July results to the present case leads to the expectation that if the belt of October 26 contained predominantly fission betas, then the VLF disturbance would, for example, have had an onset and a maximum respectively 2 and $6 \mathrm{~min}$ after the burst. This is contrary to the observation that the burst-related VLF perturbation has an onset and maximum respectively at 10 and about $30 \mathrm{~min}$ after the burst. The current elimination of fission betas from the radiation belt of October 26, 1962 expands an earlier similar suggestion resulting from the analysis of the VLF disturbance along the path from NPM in Hawaii to Anchorage, Alaska [Zmuda, Shaw, and Haave, 1963b].

\section{Nuclear Detonation of October 20, 1962}

At approximately 0830 U'T on October 20, 1962 a low-yield range device was detonated below the ionosphere at an altitude of tens of kilometers in the vicinity of Johnston Island (AGIWARN message of October 20, 1962). The detonation produced phase perturbations of several of the VLF transmissions which are normally monitored at APL and Anchorage, Alaska. The solid curves in figure 6 show for the period around the burst time, the changes in signal arrival-time for various propagation paths. For comparison, the dashed curves in this figure show the quiet-day variations for a comparable period, with the dotted portions indicating the periods of normal, scheduled interruptions of transmissions. It is relevant to note that, in data not shown here, there were no burst-related perturbations of the VLF signal from WWVL in Boulder, Colo., as received at APL.

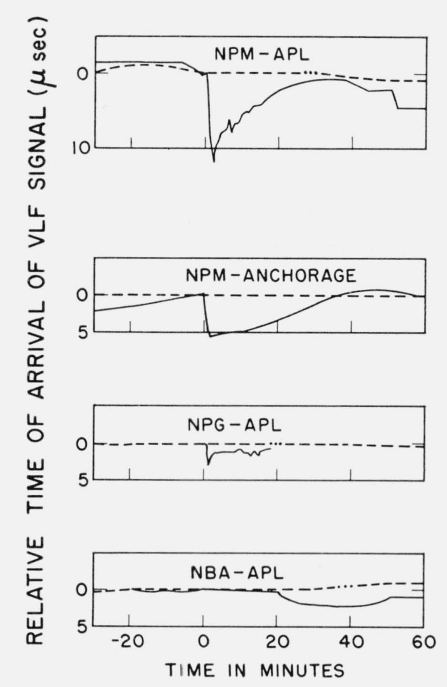

Figure 6. VLF perturbations related to the nuclear detonation of 0830 UT on October 20, 1962.

The solid curves show the variations for a period around burst-time, $t=0$; the dashed curves show quiet-day variations for a comparable period.
The perturbations of the NPM transmission as received at both APL and Anchorage start essentially simultaneously with the detonation, reach maximal deviations 2 min after the burst and then slowly recover to their preburst levels. In these disturbances there are no clear-cut indications of any delayed VLF perturbations ascribable to trapped $\beta$-rays, although the maximum deviation at $t=2$ and the subsequent slow recovery is suggestive of effects due to trapped radiation. Undoubtedly, ionization in the vicinity of NPM produced by electromagnetic and untrapped-particle emission from the burst contribute in large measure to these disturbances. The NPG-APL data pertinent to the detonation were recorded only during the period $-1<t<17 \mathrm{~min}$ but with instrumentation whose time resolution was 0.2 sec, in contrast to the other data, where a time resolution of about a minute exists. The NPG-APL perturbation (occurring on a path displaced by 5,500 $\mathrm{km}$ from the burst region) is small but readily discernible. It begins practically simultaneously with the burst and reaches a maximum about $0.6 \mathrm{~min}$ after the burst. The small, practically instantaneous, pulse of the VLF perturbation probably stems from essentially-untrapped $\beta$-rays carried to the remote VLF region by neutrons, as in the model of Crain and Tamarkin [1961].

The perturbation of the NBA transmission is so small that it ordinarily would be overlooked and, in any circumstance, cannot be taken alone as indicative of burst-related phenomena. However, it is worth noting that the disturbance has temporal characteristics totally compatible with the idea that some trapped neutron-decay betas drift from Johnston Island to the region near the NBA terminal, 9,600 $\mathrm{km}$ distant, to produce the slight VLF perturbations. For example, with the energy and drift characteristics previously discussed, the NBA-APL disturbance has an onset 20 min after the burst, a time corresponding to the drift time from the burst region to NBA of $0.78 \mathrm{Mev}$ betas, the endpoint-energy $\beta$-ray from neutron decay. In addition, the subsequent increase, broad maximum, and ultimate recovery of the VLF perturbations occur in periods generally consistent with those expected from a stream of drifting $\beta$-rays, as shown in the overhead energy curve for NBA in figure $5 \mathrm{~b}$.

\section{Nuclear Detonation of November 1, 1962}

At 1210 UT on November 1, 1962, a submegaton yield range device was detonated at an altitude of tens of kilometers in the vicinity of Johnston Island in the Pacific (AGIWARN message of November 1, 1962). The explosion produced the VLF variations shown in the solid curves of figure 7 ; for comparison, the dashed curves represent the VLF changes for a comparable period but on an adjacent quiet day. Both sets of curves are normalized with respect to the value existing at 1210 UT in their respective dates, a time taken as $t=0$.

For the NPM transmission as received at both APL and Anchorage, and for the NPG signal as 


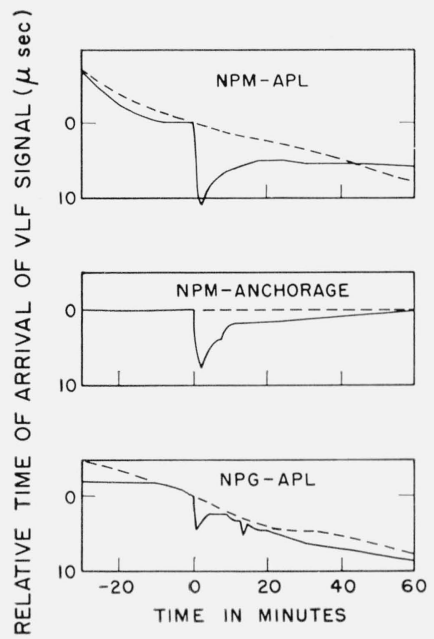

FIGURE 7. VLF perturbations related to the nuclear detonation of 1210 UT on November 1, 1962.

The solid curves show the variations for a period around burst-time, $t=0$; the dashed curves show quiet-day variations for a comparable period.

received at APL, the VLF disturbances for this event generally resemble (although of shorter duration) those for October 20 and probably have similar origins. There are no significant perturbations of the WWVL and NBA signals received at APL; these latter data are not reproduced here but it is worth noting that the sunlight contribution probably obscured the nuclear effects over the WWVL-APL and NBA-APL paths.

We gratefully acknowledge the assistance of W. E. Ray and F. H. Swaim, both of Applied Physies Laboratory, The Johns Hopkins University, Silver Spring, Md.

\section{References}

Alfvén, H. (1950), Cosmical Electrodynamies (Clarendon Press, Oxford, Eng.).

Blackband, W. T. (1963), Procedings of AGARD Meeting, Munich, Sept. 1962 (Pergamon Press, Oxford, Eng.), in press.

Brown, W. L., W. N. Hess, and J. A. Van Allen (1963), Collected papers on the artificial radiation belt from the July 9, 1962 nuclear detonation, J. Geophys. Res. 68, No. 3, 605-606.
Crain, C. M., and P. Tamarkin (1961), A note on the cause o sudden ionization anomalies in regions remote from high altitude nuclear bursts, J. Geophys. Res. 66, No. 1, 35

Evans, R. D. (1955), The Atomic Nucleus (MeGraw-Hill Book Co., Inc., New York, N.Y.)

Glasstone, S. (1962), The Effects of Nuclear Weapons, revised edition (United States Atomic Energy Commission).

Haave, C. R., A. J. Zmuda, and B. W. Shaw (1962), VLF phase perturbations associated with high-altitude nuclear bursts, APL Technical Digest $\boldsymbol{2}, 14$.

Hamlin, D. A., R. Karplus, R. C. Vik, and K. M. Watson (1961), Mirror and azimuthal drift frequencies for geomagnetically trapped particles, J. Geophys. Res. 66, No. 1, 1.

Jean, A. G., and D. D. Crombie (1963), Detection of high altitude nuclear detonations using the VLF phase shift technique, IEEE Trans on Nuclear Science, NS-10, No. 1, 242.

Latter, R., R. F. Herbst, and K. M. Watson (1961), Detecttion of nuclear explosions, Annual Review of Nuclear Science, 11, 371 (Annual Reviews, Inc., Palo Alto, Calif.).

MeIlwain, C. E. (1961), Coordinates for mapping the distribution of magnetically trapped particles, J. Geophys. Res. 66, No. 11, 3681-3691.

O'Brien, B. J., C. D. Laughlin, and J. A. Van Allen (1961), Geomagnetically trapped radiation produced by a highaltitude nuclear explosion on July 9, 1962, Nature 195, 939.

Pierce, J. A. (1955), The diurnal carrier-phase variation of a 16-kilocycle transatlantic signal, Proc. IRE 43, 584-588.

Riedler, W.. A Egeland, R. Lindquist, and A. Pedersen (1963), Effects of nuclear explosion on very-low frequency and low frequency propagation, Nature 198, 1076.

Shaw, B. W., and C. R. Haave (1962), Frequency monitoring of VLF transmission, APL Technicaı Digest, 1, 7.

Spitzer, L. Jr. (1956), Physies of Fully Ionized Gases (Interscience Publishers, New York, N.Y.).

Wait, J. R. (1959), Diurnal change of ionospheric heights deduced from phase velocity measuremets at VLF, Proc. IRE 5\%, 998.

Wait, J. R. (1963), A note on diurnal phase changes of verylow-frequency waves for long paths, J. Geophys. Res. 68, No. 1, 338-341.

Willard, H. R., and J. F. Kenny (1963), Ionospheric effects of high-altitude nuclear tests, J. Geophys. Res. 68, No. 7, $2053-2056$.

Woodward, R. H., and G. J. Gassmann (1963), Proceedings of the AGARD Meeting, Munich, Sept. 1962 (Pergamon Press Inc., New York, N.Y.), in press.

Zmuda, A. J., and B. W. Shaw, and C. R. Haave (1963a), Very low frequency disturbances and the high-altitude nuclear explosion of July 9, 1962, J. Geophys. Res. 68, No. 3, 745 .

Zmuda, A. J., B. W. Shaw, and C. R. Haave (1963b), VLF disturbances caused by the nuclear detonation of Oct. 26, 1962, J. Geophys. Res. 68, No. 13, 4105-4114.

(Paper 68D1-325) 